\title{
Untypische Verläufe sind gar nicht so selten
}

\author{
Auch bei anfänglich normaler, forcierter exspiratorischer \\ Sekundenkapazität $\left(\mathrm{FEV}_{1}\right)$ kann sich eine chronisch obstruktive \\ Lungenerkrankung (COPD) entwickeln. Andererseits indiziert \\ eine im frühen Erwachsenenalter reduzierte $\mathrm{FEV}_{1}$ nicht \\ zwangsläufig die Entwicklung einer COPD, wie eine \\ epidemiologische Analyse von P. Lange et al. aufzeigt. \\ N Engl J Med 2015; 373: 111-122
}

Die Gefahr eine COPD zu entwickeln wird mit einem reduzierten $\mathrm{FEV}_{1}$-Wert und progredienter Abnahme dieses Parameters assoziiert. Eine Definition, die bisher noch keiner epidemiologischen Überprüfung unterzogen wurde. Denkbar sind auch Verläufe mit initial unauffälligem $\mathrm{FEV}_{1}$-Befund oder nur geringer Zunahme der Atemwegsobstruktion. Außerdem bleibt unklar, welche prognostische Aussage ein, im mittleren Lebensalter erniedrigter, $\mathrm{FEV}_{1}$-Wert in Bezug auf die Ausbildung einer COPD zulässt.

Unter dem Aspekt der Krankheitsprogression bei COPD werteten die Autoren die Daten von 3 Langzeitstudien aus (Framingham Offspring Cohort, Copenhagen City Heart Study und Lovelace Smokers Cohort). So konnte der prädiktive Wert der initialen $\mathrm{FEV}_{1}$-Werte in Bezug auf eine spätere COPD aufgezeigt werden. Die $\mathrm{FEV}_{1}$-Messung zu Studienbeginn kategorisierten die Autoren als reduziert ( $<80 \%)$ oder nicht reduziert (>80\%).

Dann korrelierten die Autoren die Initialbefunde mit den Inzidenzraten der bei Studienende verifizierten COPD-Erkrankungen. Zum Studienbeginn hatten die Probanden ein Durchschnittsalter von $<40$ Jahren. Der mittlere Beobachtungszeitraum betrug 22 Jahre. Außerdem ermittelten sie, wie die einzelnen Konstellationen sich auf die durchschnittliche jährliche Abnahme der FEV ${ }_{1}$ auswirkte.

\section{Zusammenhang mit Nikotinstatus}

Bei 78\% der Patienten lag der Obstruktionsparameter initial in der Norm. Diese Probanden entwickelten zu 7\% eine COPD.
Ausschreibung

\section{Stipendium für klinische Pneumologie}

Bis zum 15. Januar 2016 haben junge Wissenschaftler erneut die Chance, sich um das mit $10000 €$ ausgestattete und von GlaxoSmithKline, München, gestiftete Forschungsstipendium für klinische Pneumologie zu bewerben. Mit diesem Stipendium möchte das Pharmaunternehmen Glaxo SmithKline zusammen mit der Deutschen Atemwegsliga e.V. den wissenschaftlichen Nachwuchs in der Pneumologie fördern.

Wenn Sie sich um das Stipendium bewerben möchten, senden Sie bitte folgende Unterlagen per Post an die Geschäftsstelle der Deutschen Atemwegsliga e.V. Raiffeisenstraße 38 in 33175 Bad Lippspringe: - einen wissenschaftlichen Lebenslauf,

- die Beschreibung des Projekts (maximal 5 DIN-A4-Seiten),

- eine Aufstellung der Projektkosten (der Anteil der Sachkosten darf maximal $50 \%$ betragen),

- eine Liste der Institutionen, bei denen ebenfalls ein Antrag auf Unterstützung des Projekts und/oder Teilprojekts gestellt wurde und

- die Bestätigung des Klinik-/Institutsleiters, dass die für die Durchführung des Forschungsvorhabens notwendigen Voraussetzungen erfüllt sind.

Teilnahmeberechtigt sind approbierte Ärzte bzw. junge Wissenschaftler mit einer vergleichbaren naturwissenschaftlichen Qualifikation, z.B. Pharmazeuten oder Biochemiker. Die Altersgrenze liegt bei 35 Jahren. Die Stipendiaten müssen einer Klinik oder einem Institut in Deutschland angehören und dürfen nicht gleichzeitig bereits durch eine andere Institution für das entsprechende Vorhaben gefördert werden. Es werden nur Projekte mit einem klar erkennbaren klinischen Bezug gefördert. Projekte der Grundlagenforschung oder reine Laborarbeiten können nicht berücksichtigt werden. Über die Vergabe des Stipendiums entscheidet ein wissenschaftlicher Beirat.

Weitere Auskünfte zum Bewerbungsverfahren gibt Dr. Uta Butt per E-Mail: uta.butt@atemwegsliga.de.

Nach einer Mitteilung der Deutschen Atemwegsliga e. V., Bad Lippspringe 